\title{
Corrigendum
}

\section{Apomorphine Enhances Conditioned Responses Induced by Aversive Stimulation of the Inferior Colliculus}

Ana Cristina Troncoso', Mario Yukio Osaki', Sueli Masson', Karina G Borelli' and Marcus Lira Brandão'

Neuropsychopharmacology (2003) 28, I016. doi:I0.1038/sj.npp.I300199

Correction to: Neuropsychopharmacology (2003) 28, 284291. doi: 10.1038/sj.npp.1300034

The authors wish to apologize that the third author name was spelled incorrectly. The correct spelling is given above. 